\title{
Foot Care Practices And Its Associated Factors among Type 2 Diabetes Patients attending Health Care Clinics in Kuantan
}

\author{
Abdul Hadi $\mathrm{A}^{1}$, Azmi $\mathrm{NH}^{1}$, Md Aris $\mathrm{MA}^{1}$, Nasreen $\mathrm{EH}^{2}$, Che Ahmad $\mathrm{A}^{3}$ \\ ${ }^{1}$ Department of Family Medicine, International Islamic University of Malaysia, \\ Kuantan, Pahang, Malaysia \\ ${ }^{2}$ Department of Orthopaedic, International Islamic University of Malaysia Kuantan \\ ${ }^{3}$ Department of Community Medicine, International Islamic University of Malaysia \\ Kuantan, Pahang, Malaysia
}

Introduction: Diabetes patients are predisposed to several micro as well as macrovascular complications. One most important and debilitating complication of diabetes are foot problems. However, it is preventable by simple intervention such as foot screening and foot care education to detect early problem so that early intervention can be done. This study aims to assess foot care practice among Type 2 diabetes patient attending primary health clinics in Kuantan. Materials and method: This was a cross-sectional study conducted at four primary health clinics in Kuantan involving 450 study participants who were selected by using convenient sampling method. Level of awareness and practice towards diabetic foot care was assessed using validated self-administered questionnaire. Multiple logistic regressions were performed to identify factors associated with foot care practice among the respondents. Results: Mean age of respondents involved was 56.36 $(S D \pm 10.9)$ years. About $59.6 \%$ of respondents had poor foot care practice. Multivariate logistic regression analysis identified that, increasing age (aOR 0.97, 95\% Cl: 0.955-0.993) and good awareness towards foot problem (aOR 0.43, 95\% Cl: 0.289-0.643) were associated with good foot care practice. However, Malay patients (aOR 1.81, 95\% Cl: 1.002-3.271) and obese patients (aOR 1.9, 95\% Cl: 1.225-2.976) were associated with poor foot care practice after controlling other variables. Other factors such as education level, diabetes control as well as overweight did not show any significant association with foot care practice. Conclusion: Based on the result, majority of respondents had poor foot care practice. Poor awareness was associated with poor foot care practice. It can be concluded that sustainable patient education and compliance towards foot care practice at primary care level should be emphasized more to ensure good foot care practice implementation. 